\section{RMD Open}

Rheumatic \&

Musculoskeletal Diseases

\title{
Variants of genes implicated in type 1 interferon pathway and B-cell activation modulate the EULAR response to rituximab at 24 weeks in rheumatoid arthritis
}

To cite: Juge P-A, Gazal S, Constantin A, et al. Variants of genes implicated in type 1 interferon pathway and B-cell activation modulate the EULAR response to rituximab at 24 weeks in rheumatoid arthritis. RMD Open 2017;3:e000448. doi:10.1136/ rmdopen-2017-000448

- Prepublication history for this paper is available online. To view these files please visit the journal online (http://dx.doi. org/10.1136/rmdopen-2017000448).

Received 1 February 2017 Revised 13 September 2017 Accepted 14 September 2017

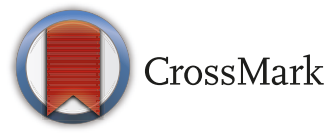

For numbered affiliations see end of article.

Correspondence to Pr Philippe Dieudé; philippe.dieude@aphp.fr

\section{ABSTRACT}

Background The type 1 interferon (IFN) pathway has been identified to potentially affect the response to rituximab (RTX) for rheumatoid arthritis (RA), which suggests the contribution of type 1 IFN pathway genes such as IFN regulatory factor 5 and 7 (IRF5 and IRF7), tyrosine kinase 2 (TYK2), signal transducer and activator of transcription 4 (STAT4) and osteopontin (SPP1). Our objective was to study functional variants of these IFN pathway genes as predictors of the European League Against Rheumatism (EULAR) response to RTX for RA at week 24 (W24).

Methods Logistic regression analysis with a stepwise multivariate model adjusted for sex, age and DAS28-CRP (Disease Activity Score in 28 joints with $C$ reactive protein) in 115 patients from the SMART randomised studywas used to analyse the association between the candidate variants and W24 EULAR response. Because the variant TNFSF13B rs9514828 was previously found associated with RTX response in the same population, it was included in the analysis.

Results The combination of IRF5 rs2004640, SPP1 rs9138 and TNFSF13B rs9514828 was strongly associated with good/moderate EULAR response to RTX at W24: $\mathrm{p}=9.34 \times 10^{-6}$, OR 11.37 (95\% Cl 4.03 to 35.28 ), positive predictive value $91 \%$ and negative predictive value $54 \%$. Conclusion Our results support the contribution of the IRF5, SPP1 and TNFSF13B genotypic combination in the response to RTX for RA at W24.

\section{BACKGROUND}

Rheumatoid arthritis (RA) is a complex systemic autoimmune disorder with environmental, genetic and stochastic factors implicated. ${ }^{1}$ The anti-CD20 chimeric monoclonal antibody that targets B cells, rituximab (RTX), is an effective treatment for RA refractory to tumour necrosis factor blockers. ${ }^{2}$ Currently, we cannot offer personalised medicine for patients with RA receiving biologics, including RTX, because we cannot identify those who

\section{Key messages}

What is already known about this subject?

- Type 1 interferon (IFN) pathway has been identified to potentially affect the rituximab (RTX) response in rheumatoid arthritis (RA).

What does this study add?

- Our results suggest an impact of genetic variations of IRF5 and SPP1, involved in the type 1 IFN pathway, on the response to RTX in RA at week 24 (W24)

- In this study, we found a strong association of the allelic combination of IRF5 rs2004640, SPP1 rs9138 and TNFSF13B rs9514828 and response to RTX in RA at W24. This finding requires replication in independent populations to further definitely conclude that the IRF5-SPP1-TNSF13B risk allelic combination is a strong predictive marker of the $\mathrm{RTX}$ response in $\mathrm{RA}$.

How might this impact on clinical practice?

- If replicated, exploring this allelic combination before the introduction of RTX could be useful for a personalised therapeutic strategy in RA.

will not respond to a given treatment, nor do we understand the pathogenesis underlying these interindividual variances. ${ }^{3}$ To improve this situation, the identification of prognostic factors of RTX response is needed.

The type 1 interferon (IFN) pathway was recently identified to potentially affect the RTX response in RA. Indeed, transcriptome studies found that the only distinction between RTX responders and non-responders at week 24 (W24) was pharmacodynamic changes in the expression of a selective group of genes all regulated by type 1 IFN. $^{45}$ As well, the pharmacological effect of RTX on 
IFN-response gene activity was found associated with IFN-response activity before the start of treatment. ${ }^{56}$

Several genes encoding proteins that are key components of the type 1 IFN system include IFN regulatory factor 5 and 7 (IRF5/7), tyrosine kinase 2 (TYK2), signal transducer and activator of transcription 4 (STAT4) and osteopontin (SPP1). These genes have recently been found to play a role in the risk of several autoimmune diseases including RA. ${ }^{7-9}$ Here, we aimed to investigate whether putative or proven functional variants of $I R F 5 / 7$, TYK2, STAT4 and SPP1 could modulate the European League Against Rheumatism (EULAR) response to RTX in RA.

\section{METHODS}

\section{Study population}

In this retrospective, multicentric study, all patients from the SMART study (AStudy of Re-treatment With MabThera (Rituximab) in Patients With Rheumatoid Arthritis Who Have Failed on Anti-TNF Alfa Therapy) who consented to genetic studies were included. Characteristics of the SMART study have been previously described. ${ }^{10}$ Briefly, 224 patients fulfilling the 1987 American College of Rheumatology criteria for RA with inadequate response, intolerance or contraindication to anti-TNFQ were included in the SMART study (NCT01126541), a 2-year national multicentric randomised open-label study evaluating the efficacy and safety of two doses of RTX for re-treatment after one course of RTX $(1000 \mathrm{mg}$ on days 1 and 15). Only corticosteroids and non-steroidal anti-inflammatory drugs were permitted if at stable doses. B-cell activating factor (BAFF) level was measured at RTX initiation using a Quantikine ELISA (R\&D Systems). The main SMART study was approved by the local ethics committee (Groupe Hospitalier Pitié-Salpêtrière, Paris), and all patients gave their informed consent according to the Declaration of Helsinki.

\section{Selection of single-nucleotide polymorphisms (SNPS)}

All patients underwent genotyping for the SNPs for IRF5 (rs2004640), IRF7 (rs1131665), STAT4 (rs7574865), TYK2 (rs2304256, rs280519 and rs12720356) and SPP1 (rs11439060 and rs9138). Genotyping was performed at KBiosciences, Manchester, UK. We selected these eight variants by their previously reported putative or proven functional relevance in the type $1 \mathrm{IFN}$ pathway. ${ }^{7-9}$ Because of the recently reported association between TNFSF13B (encoding BAFF) rs9514828 and RTX response in the SMART study ${ }^{11}$ this variant was also integrated into the multivariate analysis.

\section{Statistical analysis}

The association between the variants and EULAR response to RTX was analysed by logistic regression. ${ }^{12}$ Responders were defined by a good/moderate EULAR response at W24. First, response rate was compared across risk genotypes by univariate analysis with the $\chi^{2}$, with Yates' continuity correction as appropriate. Both dominant and recessive models were tested, and results of the most significant one were reported. Note that the additive model was also tested but results were not reported, as it did not improve the results. Variables significant at $\mathrm{p}<0.20$ were selected, combined with TNFSF13B rs9514828 and entered into a logistic regression adjusted on sex, age and Disease Activity Score in 28 joints with $\mathrm{C}$ reactive protein (DAS28-CRP), rheumatoid factor (RF) and anti-Cyclic Citrullinated Peptide (CCP) status. The best fitting model was determined using the backward procedure implemented in the step function of R. ORs and 95\% CIs were calculated. Conformity with Hardy-Weinberg equilibrium was tested by a classical $\chi^{2}$

Table 1 Overall characteristics of patients with rheumatoid arthritis by EULAR response to rituximab at week 24 (W24)

\begin{tabular}{lll}
$\begin{array}{l}\text { Overall } \\
\text { population }(\mathbf{n}=\mathbf{1 1 5})\end{array}$ & $\begin{array}{l}\text { Good/moderate } \\
\text { responders }(\mathbf{n = 9 2})\end{array}$ & Non-responders $(\mathbf{n}=\mathbf{2 2})$ \\
\hline $92(80.0)$ & $73(79.3)$ & $18(81.8)$ \\
$55.50 \pm 11.15$ & $55.47 \pm 11.33$ & $55.77 \pm 10.84$ \\
$14.06 \pm 9.29$ & $14.07 \pm 9.55$ & $14.25 \pm 8.54$ \\
$93(80.9)$ & $75(81.5)$ & $17(77.3)$ \\
$82(71.3)$ & $68(73.9)$ & $13(59.1)$ \\
$87(75.7)$ & $66(71.7)$ & $20(90.9)$ \\
$7.72 \pm 2.90$ & $7.64 \pm 2.90$ & $8.02 \pm 2.68$ \\
& & \\
$5.68 \pm 0.90$ & $5.76 \pm 0.87$ & $5.34 \pm 0.97$ \\
$3.94 \pm 1.07$ & $3.63 \pm 0.84$ & $5.23 \pm 0.98$ \\
$-1.74 \pm 1.14$ & $-2.13 \pm 0.87$ & $-0.11 \pm 0.55$ \\
$588.34 \pm 364.51$ & $590.89 \pm 368.23$ & $475.8 \pm 50.20$
\end{tabular}

DAS28-CRP, Disease Activity Score in 28 joints with C reactive protein; EULAR, European League Against Rheumatism; RF, rheumatoid factor; RTX, rituximab. 
test with one degree of freedom in controls. All statistical analyses involved the use of $R$ V.3.1.2. p-value $<0.05$ was considered statistically significant.

\section{RESULTS}

Among the 224 patients with RA included in the SMART study, 115 gave consent for genetic analysis and their data were analysed in this study; 92 were women $(80 \%)$, mean age was $55.50 \pm 11.15$ years, 93 had anti-CCP antibodies $(80.9 \%)$ and 82 were positive for RF $(71.3 \%)$. Mean DAS28-CRP was $5.68 \pm 0.90$ at RTX initiation and $3.94 \pm 1.07$ at W24, with a mean change of $-1.74 \pm 1.14$. At W24, 30 (26.3\%) patients were considered good responders, $62(54.4 \%)$ moderate responders and 22 $(19.3 \%)$ non-responders (table 1). Genotype frequencies were in Hardy-Weinberg equilibrium for all SNPs investigated (data not shown). Genotyping results are in table 2.
Univariate analysis revealed an increase in the minor allele frequency for $I R F 5$ rs2004640, IRF7 rs1131665 and SPP1 rs9138 in good/moderate responders at W24 (ie, $\mathrm{p}<0.20$, table 2). To further define the model best fitting a EULAR response to RTX at W24, we added the variant TNFSF13B rs9514828 ${ }^{11}$ in the multivariate analysis.

Multivariate analysis revealed an allelic combination of the three distinct variants $I R F 5$ rs2004640, SPPI rs9138 and TNFSF13B rs9514828 strongly associated with EULAR response to RTX at W24, independent of the anti-citrullinated protein antibodies (ACPA) status of the patients, defined by homozygosity for the common allele of SPP1 rs9138 (genotype A/A) and TNFSF13B rs9514828 (genotype $\mathrm{C} / \mathrm{C}$ ) and carrying at least one rare allele of IRF5 rs2004640 (genotype G/T or G/G). This predictive allelic combination was found in 69 of good/ moderate responders $(75.0 \%)$ compared with seven

Table 2 Univariate analysis of good/moderate EULAR response at W24 by genotype

\begin{tabular}{|c|c|c|c|c|c|}
\hline Candidate gene & Candidate variants & Genotype* & $\begin{array}{l}\text { Good/moderate } \\
\text { responders }\end{array}$ & Non-responders & p Value \\
\hline \multirow[t]{3}{*}{ IRF5 } & rs2004640 & $0(\mathrm{~T} / \mathrm{T})$ & 17 & 10 & \\
\hline & & $1(\mathrm{~T} / \mathrm{G})$ & 56 & 8 & $0.030 \ddagger$ \\
\hline & & $2(G / G)$ & 12 & 4 & \\
\hline \multirow[t]{3}{*}{ IRF7 } & rs1131665 & $0(\mathrm{~T} / \mathrm{T})$ & 45 & 15 & \\
\hline & & $1(\mathrm{~T} / \mathrm{C})$ & 35 & 6 & $0.180 \dagger$ \\
\hline & & $2(\mathrm{C} / \mathrm{C})$ & 11 & 1 & \\
\hline \multirow[t]{6}{*}{ SPP1 } & rs11439060 & $0(-/-)$ & 48 & 13 & \\
\hline & & $1(-/ G)$ & 30 & 7 & \\
\hline & & $2(G G)$ & 13 & 1 & 0.410 \\
\hline & rs9138 & $0(\mathrm{~A} / \mathrm{A})$ & 45 & 6 & \\
\hline & & $1(\mathrm{C} / \mathrm{A})$ & 33 & 12 & $0.085 \dagger$ \\
\hline & & $2(\mathrm{C} / \mathrm{C})$ & 11 & 4 & \\
\hline \multirow[t]{3}{*}{ STAT4 } & rs7574865 & $0(\mathrm{G} / \mathrm{G})$ & 41 & 12 & \\
\hline & & $1(\mathrm{G} / \mathrm{T})$ & 42 & 6 & \\
\hline & & $2(T / T)$ & 7 & 4 & 0.284 \\
\hline \multirow[t]{3}{*}{ TNFSF13B } & rs9514828 & $0(\mathrm{C} / \mathrm{C})$ & 36 & 3 & \\
\hline & & $1(\mathrm{C} / \mathrm{T})$ & 43 & 11 & \\
\hline & & $2(\mathrm{~T} / \mathrm{T})$ & 13 & 8 & $0.035 \ddagger$ \\
\hline \multirow[t]{9}{*}{ TYK2 } & rs12720356 & $0(\mathrm{~T} / \mathrm{T})$ & 73 & 20 & \\
\hline & & $1(T / G)$ & 13 & 2 & 0.490 \\
\hline & & $2(\mathrm{G} / \mathrm{G})$ & 3 & 0 & \\
\hline & rs2304256 & $0(\mathrm{C} / \mathrm{C})$ & 45 & 8 & \\
\hline & & $1(\mathrm{C} / \mathrm{A})$ & 35 & 11 & 0.515 \\
\hline & & $2(\mathrm{~A} / \mathrm{A})$ & 8 & 1 & \\
\hline & rs280519 & $0(\mathrm{~A} / \mathrm{A})$ & 27 & 6 & \\
\hline & & $1(\mathrm{~A} / \mathrm{G})$ & 37 & 12 & \\
\hline & & $2(\mathrm{G} / \mathrm{G})$ & 28 & 4 & 0.376 \\
\hline
\end{tabular}

Grey indicates the selected model (dominant or recessive).

${ }^{*} 0$ denotes patients homozygous for the major allele; 1 , heterozygous; 2 , homozygous for the rare allele.

$\mathrm{tp}<0.20$.

$\neq \mathrm{p}<0.05$.

EULAR, European League Against Rheumatism. 
A

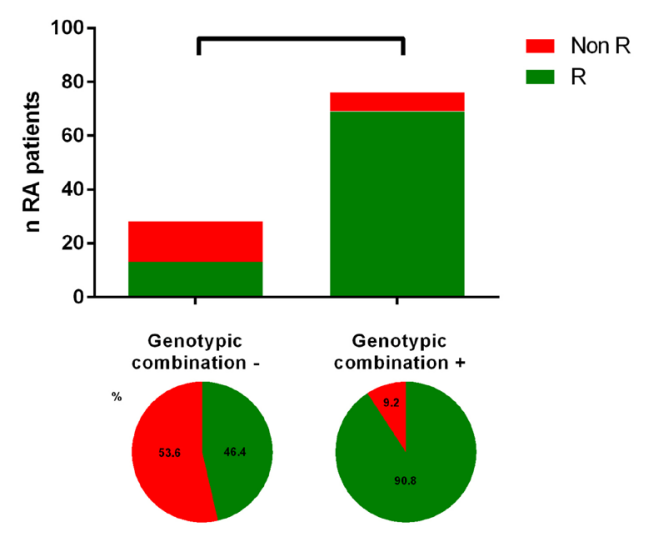

B

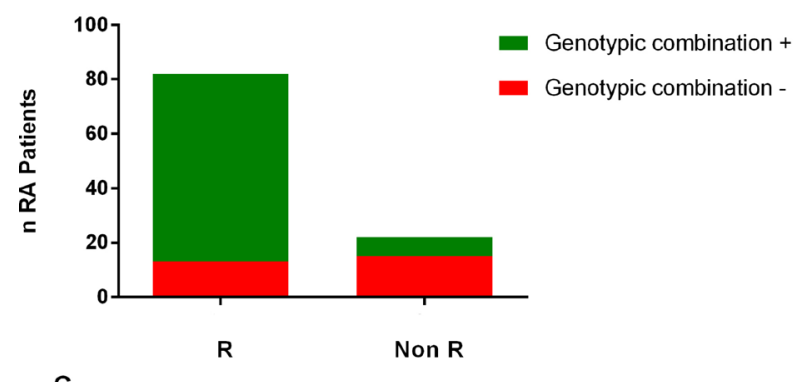

C

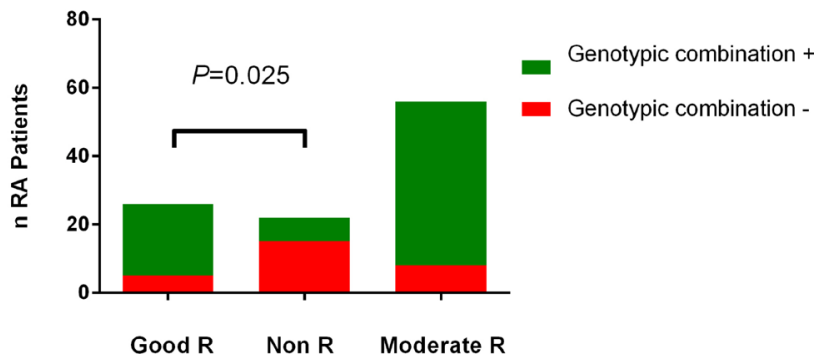

Figure 1 (A) Number and percentage of patients with rheumatoid arthritis (RA) who were European League Against Rheumatism (EULAR) responders and non-responders to rituximab by carriage of the IRF5 rs2004640, SPP1 rs9138 and TNFSF13B rs9514828 genotypic combination; (B) EULAR responders and non-responders with and without the genotypic combination. R, responders; Non R, nonresponders.

non-responders $(31.8 \%)\left(\mathrm{p}=3.27 \times 10^{-5}\right.$, OR $9.85(95 \% \mathrm{CI}$ 3.47 to 30.74$)$ ), positive predictive value $91 \%$ and negative predictive value $54 \%$ (figure 1 ). The association was still significant when good responders were compared with non-responders ( $\mathrm{p}=0.0025$, OR 6.73 (95\% CI 2.05 to 24.78)) (figure 1). The genotypic combination was not statistically associated with the BAFF level at RTX initiation and only a trend was observed $(p=0.105)$.

\section{DISCUSSION}

RA is a clinical heterogeneous disease and the therapeutic strategy is challenging because of its interindividual variability. Since the last decade, medicine has begun to embrace genomic tools that allow for more precise prediction in treatment response. However, to date, predictive tools to identify patients with RA who will respond to a given biologic remains to be identified. Recently, a type 1 IFN signature was found associated with good response to RTX in RA. ${ }^{45}$ Consequently, we investigated functional genetic variants in genes encoding proteins involved in the type 1 IFN pathway and found a strong association of the allelic combination of IRF5 rs2004640, SPP1 rs9138 and TNFSF13B rs9514828 and response to RTX at W24.

Our results suggest an impact of genetic variations of $I R F 5$ and SPP1, involved in the type 1 IFN pathway, and TNFSF13B, implicated in B-cell maturation, homoeostasis and survival, in $\mathrm{RA}^{13}$ on the response to RTX in RA. This impact might explain part of the interindividual variability in RTX response observed in RA. We recently identified SPP1 rs9138 as a new RA susceptibility variant, which was also identified as having a prognostic effect by contributing to the progression of joint damage in ACPA-negative RA. ${ }^{14}$ Our results reinforce the role of SPP1 as a modifier gene in RA by affecting the RTX response. Of interest, similar to SPP1, IRF5 has also been reported as a susceptibility and modifier gene in RA. ${ }^{15}$ To our knowledge, this is the first study providing evidence of a strong contribution of an allelic combination and RTX response in RA; the magnitude of the association between the IRF5-SPP1-TNFSF13B allelic combination is 2.8 times higher than that observed for TNFSF13B rs9514828 alone, which suggests a synergistic effect of the type 1 IFN variants. ${ }^{11}$

Our results were based on the analysis of a single population of RA cases, which is one limitation of our study; the study needs to be replicated in an independent larger population to further confirm a strong contribution of the IRF5-SPP1-TNFSF13B allelic combination in the RTX response. Given a power of detection $>0.95$, the OR of 11.37, the frequency of the IRF5-SPP1-TNFSF13B allelic combination and the rate of $20 \%$ of non-responders, a sample of 400 individuals would be required to replicate our findings.

The pooled functional consequences of the IRF5SPP1-TNFSF13B allelic combination remains to be determined; however, IRF5 rs2004640 is in complete linkage disequilibrium with a 5 bp (CGGGG insertion/deletion) promoter polymorphism found correlated with mRNA levels of IFN-induced genes, ${ }^{16}$ and SPP1 rs9138 affects osteopontin level, ${ }^{17}$ an extracellular-matrix-glycosylated phosphoprotein implicated in type I IFN production. ${ }^{18}$ In addition, the TNFSF13B rs9514828 rare allele has been found associated with increased transcription of the BAFF cytokine. ${ }^{19}$ Unfortunately, we could not detect a functional effect of the genotypic combination using the BAFF level at initiation, as the association did not reach statistical significance. This may be due to the small number of included patients as only 90 patients had a BAFF level available for the analysis. Since we could not confirm the functional effect of the combination in our study, replication in independent populations is required to support our results.

Because the genotypic combination was strongly associated to the RTX treatment efficacy, it may also affect the 
safety profile to RTX. Indeed, a better efficacy to RTX may be due to a stronger B-cell depletion, which could be correlated to more side effects in particular infectious events. Our study did not assess the safety profile of the patients, and studies with a longer follow-up should be done in order to better characterise the safety profile of the patients depending on their genotype.

\section{CONCLUSIONS}

In conclusion, our results support the contribution of IRF5, SPP1 and TNFSF13B in the response to RTX treatment in RA at W24. However, an independent replication is required to establish the exact contribution of the alleles rs2004640, rs9138 and rs9514828 in the RTX response. Identifying individual genetic risk factors would increase our understanding of the mechanisms underlying variation in response to RTX. If validated, our model could help to define a personalised strategy for patients with RA.

\author{
Author affiliations \\ ${ }^{1}$ Department of Rheumatology, Assistance Publique-Hôpitaux de Paris, Bichat \\ Hospital, Université Paris Diderot, Paris, France \\ ${ }^{2}$ Plateforme de Génomique Constitutionnelle, Assistance Publique-Hôpitaux de \\ Paris, Bichat Hospital, Université Paris Diderot, Paris, France \\ ${ }^{3}$ UMR 1027, INSERM, Toulouse III University, Toulouse, France \\ ${ }^{4}$ Department of Rheumatology, Hôpital Purpan, CHU Toulouse, Toulouse, France \\ ${ }^{5}$ Department of Rheumatology, Université Paris-Sud, Assistance Publique- \\ Hôpitaux de Paris, Paris, France \\ ${ }^{6}$ INSERM U1184, Université Paris-Sud, Paris, France \\ ${ }^{7}$ Department of Rheumatology, Service d'Immuno-Rhumatologie, Montpellier, \\ France \\ ${ }^{8}$ Department of Rheumatology, Hôpital Lyon-sud, Pierre-Bénite, France \\ ${ }^{9}$ Department of Rheumatology, Université Paris Descartes, Hôpital Cochin, \\ Assistance Publique-Hôpitaux de Paris, Paris, France \\ ${ }^{10}$ INSERM U1153, PRES Sorbonne Paris-Cité, Paris, France \\ ${ }^{11}$ Centre de Référence des Maladies Auto-immune Rares, Hôpitaux Universitaires \\ de Strasbourg, Strasbourg, France \\ ${ }^{12}$ Department of Rheumatology, Rouen University Hospital, Rouen, France \\ ${ }^{13}$ INSERM U699, Université de Paris Diderot, PRES Sorbonne Paris-Cité, Paris, \\ France
}

Acknowledgements We thank all the SMART investigators and Laura Smales (Toronto, Ontario, Canada) for editing the manuscript.

Contributors P-AJ and PD designed the study. All authors collected the patients data. SG and PD carried out the statistical analyses. P-AJ and PD wrote the first version of the manuscript. All authors read and approved the final version of the manuscript.

Funding SMART study was sponsored by ROCHE, France.

Competing interests PD is supported by an unrestricted grant from ROCHE.

Patient consent Obtained.

Ethics approval The main SMART study was approved by the local ethics committee (Groupe Hospitalier Pitié-Salpêtrière, Paris).

Provenance and peer review Not commissioned; externally peer reviewed.

Data sharing statement Data are available on demand by emailing the corresponding author.

Open Access This is an Open Access article distributed in accordance with the Creative Commons Attribution Non Commercial (CC BY-NC 4.0) license, which permits others to distribute, remix, adapt, build upon this work non-commercially, and license their derivative works on different terms, provided the original work is properly cited and the use is non-commercial. See: http://creativecommons.org/ licenses/by-nc/4.0/

(c) Article author(s) (or their employer(s) unless otherwise stated in the text of the article) 2017. All rights reserved. No commercial use is permitted unless otherwise expressly granted.

\section{REFERENCES}

1. Klareskog L, Catrina Al, Paget S. Rheumatoid arthritis. The Lancet 2009;373:659-72.

2. Edwards JC, Szczepanski L, Szechinski J, et al. Efficacy of B-celltargeted therapy with rituximab in patients with rheumatoid arthritis. N Engl J Med 2004;350:2572-81.

3. Verweij CL. Predicting the future of anti-tumor necrosis factor therapy. Arthritis Res Ther 2009;11:115.

4. Vosslamber S, Raterman HG, van der Pouw Kraan TC, et al. Pharmacological induction of interferon type I activity following treatment with rituximab determines clinical response in rheumatoid arthritis. Ann Rheum Dis 2011;70:1153-9.

5. van der Pouw Kraan TC, Wijbrandts CA, van Baarsen LG, et al. Rheumatoid arthritis subtypes identified by genomic profiling of peripheral blood cells: assignment of a type I interferon signature in a subpopulation of patients. Ann Rheum Dis 2007;66:1008-14.

6. Thurlings RM, Boumans M, Tekstra J, et al. Relationship between the type I interferon signature and the response to rituximab in rheumatoid arthritis patients. Arthritis Rheum 2010;62:3607-14.

7. Kariuki SN, Moore JG, Kirou KA, et al. Age- and gender-specific modulation of serum osteopontin and interferon-alpha by osteopontin genotype in systemic lupus erythematosus. Genes Immun 2009;10:487-94.

8. Fu Q, Zhao J, Qian X, et al. Association of a functional IRF7 variant with systemic lupus erythematosus. Arthritis Rheum 2011;63:749-54.

9. Okada Y, Wu D, Trynka G, et al. Genetics of rheumatoid arthritis contributes to biology and drug discovery. Nature 2014;506:376-81.

10. Mariette X, Rouanet S, Sibilia J, et al. Evaluation of low-dose rituximab for the retreatment of patients with active rheumatoid arthritis: a non-inferiority randomised controlled trial. Ann Rheum Dis 2014;73:1508-14.

11. Ruyssen-Witrand $A$, Rouanet $S$, Combe B, et al. Association between $-871 \mathrm{C}>\mathrm{T}$ promoter polymorphism in the B-cell activating factor gene and the response to rituximab in rheumatoid arthritis patients. Rheumatology 2013;52:636-41.

12. van Gestel AM, Haagsma CJ, van Riel PL. Validation of rheumatoid arthritis improvement criteria that include simplified joint counts. Arthritis Rheum 1998:41:1845-50.

13. Nakajima K, Itoh $\mathrm{K}$, Nagatani $\mathrm{K}$, et al. Expression of BAFF and BAFF-R in the synovial tissue of patients with rheumatoid arthritis. Scand J Rheumatol 2007;36:365-72.

14. Juge PA, van Steenbergen HW, Constantin A, et al. SPP1 rs9138 variant contributes to the severity of radiological damage in anticitrullinated protein autoantibody-negative rheumatoid arthritis. Ann Rheum Dis 2014;73:1840-3.

15. Dawidowicz K, Allanore Y, Guedj M, et al. The interferon regulatory factor 5 gene confers susceptibility to rheumatoid arthritis and influences its erosive phenotype. Ann Rheum Dis 2011;70:117-21.

16. Miceli-Richard C, Gestermann N, Ittah M, et al. The CGGGG insertion/deletion polymorphism of the IRF5 promoter is a strong risk factor for primary Sjögren's syndrome. Arthritis Rheum 2009;60:1991-7.

17. Chiocchetti A, Indelicato M, Bensi T, et al. High levels of osteopontin associated with polymorphisms in its gene are a risk factor for development of autoimmunity/lymphoproliferation. Blood 2004;103:1376-82.

18. Shinohara ML, Lu L, Bu J, et al. Osteopontin expression is essential for interferon-alpha production by plasmacytoid dendritic cells. Nat Immunol 2006;7:498-506.

19. Nossent JC, Lester S, Zahra D, et al. Polymorphism in the 5' regulatory region of the $B$-lymphocyte activating factor gene is associated with the Ro/La autoantibody response and serum BAFF levels in primary Sjogren's syndrome. Rheumatology 2008;47:1311-6. 